\title{
Encrusted cystitis after definitive radiotherapy for cervical cancer: a case report
}

\author{
Elisabetta Perrucci, MD', Valentina Lancellotta, MD², Maika di Benedetto, MD³, Isabella Palumbo, MD², \\ Fabio Matrone, MD³, Marino Chiodi, MD4, Riccardo Lombi, MD5, Marta Marcantonini, BSc ${ }^{6}$, Cristina Mariucci, MD³, \\ Cynthia Aristei, MD2 \\ 'Radiation Oncology Section, Santa Maria della Misericordia Hospital, 2Radiation Oncology Section, University of Perugia and Santa Maria \\ della Misericordia Hospital, ${ }^{3}$ Radiation Oncology Section, University of Perugia, ${ }^{4}{ }^{2 n d}$ Radiology Section, Santa Maria della Misericordia Hospital, \\ 5Urology Section, Santa Maria della Misericordia Hospital, ‘Medical Physics Unit, Santa Maria della Misericordia Hospital, Perugia, Italy
}

\begin{abstract}
Purpose: Encrusted cystitis is a rare chronic inflammatory disease characterized by calcified plaques of the bladder, previously altered by varies conditions as urological procedures, caused by urea-splitting bacteria. Only one case has been reported on encrusted cystitis occurring after surgery and radiation therapy for a pelvic neoplasm. We report on encrusted cystitis occurred after definitive radiotherapy for bulky uterine cervix cancer, and examine the doses to the bladder wall and the procedure of radiation treatment performed as a possible cause of the onset of the disease.

Case presentation: A 52-year-old female developed encrusted cystitis, caused by Corynebacterium spp., after 14 months from definitive chemo-radiotherapy and 2/D brachytherapy treatment for FIGO stage IB2 uterine cervix cancer. For pelvic radiotherapy, the mean bladder dose was 48.47 Gy (range 31.20-51.91); maximal bladder point doses at each brachytherapy insertions were 7.62 Gy, 4.94 Gy and 6.27 Gy at first, second, and third fraction, respectively. Total biological effective dose (BED) at bladder point was $140.05 \mathrm{~Gy}_{3}$. The patient was administered antibiotic therapy with linezolid and urine acidification with vitamin C; dietary norms were also suggested. After therapy, complete remission of symptoms and radiological findings were achieved, and the planned surgery for removing the calcified plaques was not completed. After 5 years from the cervical cancer diagnosis, the patient was disease-free without urinary symptoms.

Conclusions: The high doses administered to the bladder wall and the repeated catheterizations performed at each brachytherapy insertions may have favored the infection and promoted the occurrence of the encrusted cystitis.
\end{abstract}

Key words: brachytherapy, cervical cancer, encrusted cystitis, radiotherapy.

\section{Purpose}

Encrusted cystitis (EC), first described in 1914 [1], is a chronic bladder inflammation with ulceration characterized by calcific plaques, causing troubling urinary symptoms as dysuria and gross hematuria, occurring in immunocompromised patients submitted to renal transplantation or in patients who had undergone urological procedures $[1,2,3,4,5]$. In fact, the bacteria causing the disease are implanted in a bladder wall already altered by tumor, inflammatory condition, transurethral resection, and long-term catheterization. The pathogens, most often the Corynebacterium urealyticum, which is present in the skin flora in $12 \%$ of the population, have urea-splitting activity and determine urine alkalinization [6,7]. The modification of urine $\mathrm{pH}$ favors the precipitation of urinary salts into the bladder mucosa. The characteristic encrustations of the bladder and the walls of other parts of urinary system are detected by cystoscopy, ultrasound, and radiologi- cal exams $[1,2,3,4,5,6,7]$. Until now, only the report from Parra Muntaner et al. [2] in 1996 reported on the occurrence of EC in a patient who had undergone surgery and pelvic radiotherapy (RT) for gynecological cancer. Here, we present a case of EC occurred in a patient submitted pelvic RT and brachytherapy (BT) as definitive treatment for uterine cervix cancer, and examine the procedures and the bladder dose that could have been the promoters of the disease.

\section{Case presentation}

A 52-year-old patient diagnosed with uterine cervix cancer, Federation International of Gynecology and Obstetrics (FIGO) stage IB2, was submitted to intensity modulated pelvic RT without central shield, single dose $1.8 \mathrm{~Gy}$, total dose $50.4 \mathrm{~Gy}, 5$ week fractions. Weekly concomitant chemotherapy with cisplatin, $40 \mathrm{mg} / \mathrm{m}^{2}$, for six cycles was administered, without hematologic toxicity. 


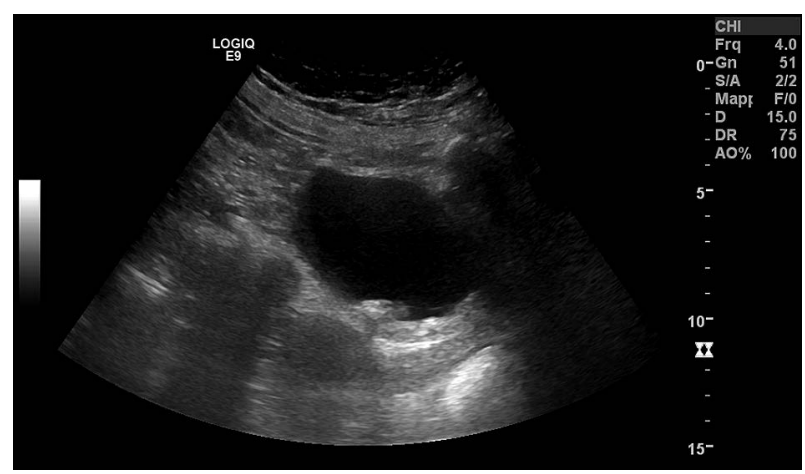

Fig. 1. Pelvic ultrasound showing thickening of the posterior bladder wall and diffuse hyperechoic plaques

On dose-volume histograms, the mean external beam bladder dose was 48.47 Gy (range 31.20-51.91). Highdose-rate (HDR) 2/D utero-vaginal BT with ${ }^{192}$ Ir source was administered at the end of pelvic RT. Doses were calculated on the orthogonal radiographic films acquired at each BT insertion according to International Commission on Radiation Units and Measurements (ICRU) 38 recommendations [8]. Single dose of 7 Gy, for 3 weekly fractions, was prescribed at point A (defined as $2 \mathrm{~cm}$ above and $2 \mathrm{~cm}$ laterally from external uterine orifice marked by radiopaque marker). Bladder doses were evaluated at each fraction by Foley catheter with iodine contrast in the balloon. Maximum bladder point doses were 7.62 Gy, 4.94 Gy, and 6.27 Gy at first, second, and third fraction, respectively. Globally, total biological effective dose (BED) at bladder point was $140.05 \mathrm{~Gy}_{3}$.

At the end of planned treatment, the patient achieved a complete clinical remission of the disease, and after 4 months, a complete pathological remission was documented by negative colposcopic examination and cervical biopsies; positron emission tomography-computed tomography (PET-TC) after 6 months and magnetic resonance imaging (MRI) after 1 year were negative.

After 14 months, the patient developed increasing dysuria and pollachyuria, firstly treated with nonspecific antibiotic therapy, without benefit. Subsequently, the patient presented hematuria, fever, and pelvic pain. Standard urine culture was negative. Pelvic ultrasound showed thickening of the posterior bladder wall and diffuse hyperechoic plaques, suspected for EC (Figure 1). Magnetic resonance imaging (Figure 2) and cystoscopy confirmed the diagnosis. Bladder biopsies showed necrotic material and inflammatory granulation tissue. The urine culture was positive for Corynebacterium simulans. On the basis of antibiogramma, the patient undertook therapy with linezolid $600 \mathrm{mg} \times 2$ /die for 1 month. Also, urine acidification with oral vitamin $\mathrm{C}$ and complementary dietary norms were suggested. After the end of antibiotic therapy, the patient achieved complete remission of symptoms, confirmed at ultrasound exam and at cystoscopy, after which the planned surgery for removing calcified plaques was not done. After 2 years, during routine follow-up, she was diagnosed with early breast cancer and underwent conservative surgery, adjuvant hypofractionated whole-breast RT, and hormonal therapy with aromatase

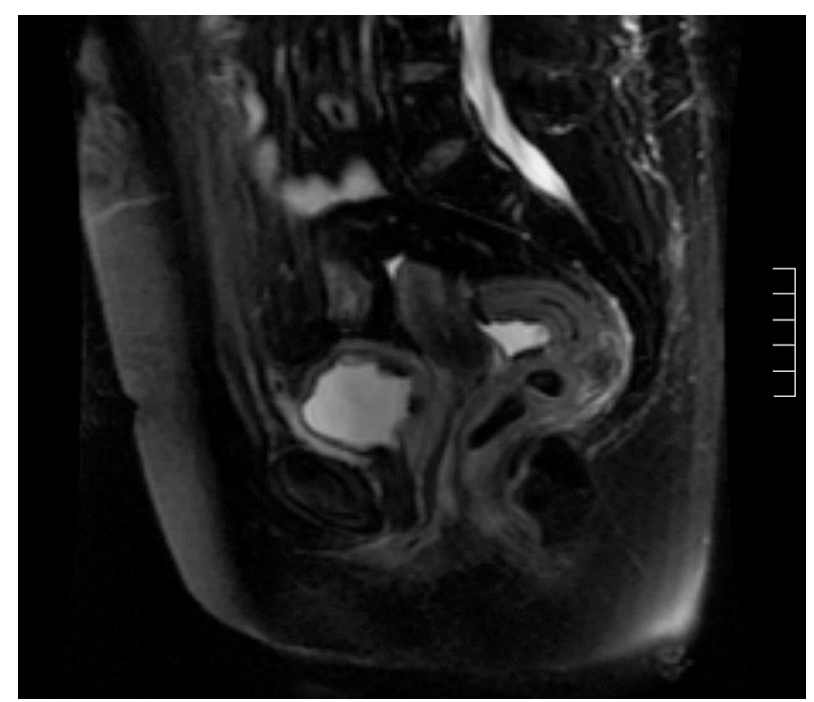

Fig. 2. Sagittal section of pelvic magnetic resonance imaging showing urinary bladder wall thickening

inhibitor. After 5 years from cervical cancer diagnosis, the patient was in complete remission without gynecological and breast cancer, free from urinary symptoms. Computed tomography (CT) scan confirmed slight thickening of the bladder and rectal walls, likely post-radiation effects.

\section{Discussion}

To our knowledge, this is the first case of EC reported after definitive RT for a pelvic cancer. In particular, Parra Muntaner et al. [2] described the occurrence of EC in a woman affected by gynecological cancer submitted surgery and adjuvant pelvic RT. No other descriptions on doses and RT modalities are known.

Our patient had the first urinary symptoms after 14 months from the end of treatment, concurring with the reported latency between urological procedures and the onset of symptoms that could range from few days to 3 years [1].

Conditions that could have promote the disease occurrence are the acute mucosa reaction due to the RT and the catheterizations performed at each BT fraction. The doses administered at the bladder wall by BT were evaluated on a 2D plan and were referred at maximum ICRU [8] bladder point. By applying the linear-quadratic model for the three HDR fractions, the EQD2 bladder doses were $16.18 \mathrm{~Gy}, 7.84 \mathrm{~Gy}$ and $11.62 \mathrm{~Gy}$, respectively. Even though the ICRU point dose could underestimate the true dose received by the organ at risk [9], and adding both the doses reached by external beam and BT treatments, we can speculate that the cumulative bladder dose was probably around $87.55 \mathrm{~Gy}$ at least at maximum dose point of the bladder wall. The cumulative BED dose, reached by pelvic RT and BT, exceeded the value of $125 \mathrm{~Gy}_{3}$, corresponding to $75 \mathrm{~Gy}$, which is reported as safe for the occurrence of late toxicity $[9,10]$. Ferrigno et al. [10] reported a higher rate of 5-year bladder complication, which was no statistically significant; in 138 patients submitted, pelvic $\mathrm{RT}$ and 2/D BT: $17 \%$ versus 9\% for BED vales over or 
below the cut-off of $125 \mathrm{~Gy}_{3}$. In our patient, no late bladder toxicity was scored, except the post-radiation thickening of the bladder wall, scored at CT and MRI follow-up examinations. Of note, urine $\mathrm{pH}$ in our patient was always not alkaline, contrary to that is generally reported [1], but the urine exams were performed after the infection was established.

Surgery was not performed in our patient, because of the complete remission of disease scored after therapy. A conservative management of the disease, excluding surgery, was also performed and it was recommended by Parra Muntaner et al. [2]. Of note, in our patient urine acidification was performed by oral administration of vitamin $\mathrm{C}$, enhanced by dietary norms; on the contrary, urine acidification is generally performed by administering local or systemic therapy.

\section{Conclusions}

The bladder wall inflammation, due to RT treatment and repeated catheterizations requested at each BT fraction, could create a fertile substrate for the rooting of germs responsible for EC. Surgery for removing calcifications could be omitted in patient reaching a complete remission of the disease after pharmacological therapy.

\section{Disclosure}

Authors report no conflict of interest.

\section{References}

1. Meria P, Desgrippes A, Arfi C et al. Encrusted cystitis and pyelitis. J Urol 1998; 160: 3-9.

2. Parra Muntaner L, Rivas Escudero JA, Gómez Cisneros S et al. Bilateral obstructive uropathy secondary to encrusted cystitis. Report of a case. Arch Esp Urol 1996; 49: 870-872.

3. Johnson MH, Strope SA. Encrusted cystitis. Urology 2012; 79: e31-e32.

4. Lieten S, Schelfaut D, Wissing KM et al. Alkaline-encrusted pyelitis and cystitis: an easily missed and life-threatening urinary infection. BMJ Case Rep 2011; 2011.

5. Favre G, Garcia-Marchiñena P, Bergero M et al. Diagnosis and treatment of the encrusted cystitis. Actas Urol Esp 2010; 34: 477-488.

6. Del Prete D, Polverino B, Ceol M et al. Encrusted cystitis by Corynebacterium urealyticum: a case report with novel insights into bladder lesions. Nephrol Dial Transplant 2008; 23: 2685-2687.

7. Soriano F, Ponte C, Santamaria M et al. Corynebacterium group D2 as a cause of alkaline-encrusted cystitis: report of four cases and characterization of the organisms. J Clin Microbiol 1985; 21: 788-792.

8. ICRU Report 38. Dose and volume specification for reporting intracavitary therapy in gynecology. International Commission on Radiation Units and Measurements, Bethesda 1985; 1-20.

9. Chen SW, Liang JA, Yeh LS et al. Comparative study of reference points by dosimetric analyses for late complications after uniform external radiotherapy and high-dose-rate brachytherapy for cervical cancer. Int J Radiat Onco Biol Phys 2004; 60: 663-671.

10. Ferrigno R, Dos Santos Novaes PER, Pelizzon AC et al. Highdose-rate brachytherapy in the treatment of uterine cervix cancer. Analysis of dose effectiveness and late complications. Int J Radiat Onco Biol Phys 2001; 50: 1123-1135. 